Revista de Matemática: Teoría y Aplicaciones 2017 24(1) : 1-7

CIMPA - UCR ISSN: 1409-2433 (PRINT), 2215-3373 (ONLINE)

\title{
SEMIRETÍCULOS Y OBJETOS K-FINITOS
}

\section{SEMILATTICES AND K-FINITE OBJECTS}

\author{
OSVALDO ACUÑA-ORTEGA*
}

Received: 10/Sep/2015; Revised: 9/Aug/2016;

Accepted: $7 / 0 c t / 2016$

Revista de Matemática: Teoría y Aplicaciones is licensed under a Creative Commons Reconocimiento-NoComercial-Compartirigual 4.0 International License.

Creado a partir de la obra en http://www.revistas.ucr.ac.cr/index.php/matematica

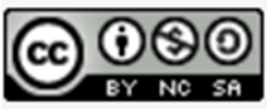

*CIMPA, Escuela de Matemática, Universidad de Costa Rica, San José, Costa Rica. E-Mail: maritzaavendanorivera@hotmail.es 


\title{
Resumen
}

Se prueba que si $X$ es un objeto de un topos elemental entonces las propiedades siguientes son equivalentes: (a) $X$ es $K$-finito, (b) para todo semiretículo con uniones binarias $B$, la diagonal $B \rightarrow B^{X}$ tiene un adjunto izquierdo.

Palabras clave: topos; objetos k-finitos; semiretículos; decidibilidad.

\begin{abstract}
We prove that for $X \in|E|, E$ elementary topos, the following properties are equivalent: (a) $X$ is $K$-finite, (b) for every upper semilattice $B$, the diagonal $B \rightarrow B^{X}$ has a left adjoint.
\end{abstract}

Keywords: topoi; k-finite objects; semilattice; decidability.

Mathematics Subject Classification: 03G30, 18B25.

\section{Introducción}

Sea $E$ un topos elemental arbitrario. Sea $(B, \leq)$ un objeto parcialmente ordenado, $B$ es completo si $\downarrow \operatorname{seg}_{B}: B \rightarrow \Omega^{B}$ tal que $\models \downarrow \operatorname{seg}_{B}(b)=\{x / x \leq b\}$ tiene un adjunto izquierdo $\sup _{B}: \Omega^{B} \rightarrow B$. Si $(B, \leq)$ es un objeto parcialmente ordenado, $F: X \rightarrow \Omega^{B}$ se dice ser dirigido si:

(a) Existe $\beta: Y \rightarrow X$ sobreyectiva y $b: Y \rightarrow B$ tal que $b \in \beta \circ F$.

(b) Para toda $\alpha: Z \rightarrow X$ y todo par $b_{1}, b_{2}: Z \rightarrow B$ tal que $b_{1} \in \alpha \circ F$ y $b_{2} \in \alpha \circ F$ existe $\beta_{1}: Z_{1} \rightarrow Z$ sobreyectiva y $b_{3}: Z_{1} \rightarrow B$ tal que $b_{3} \in \beta_{1} \circ \alpha \circ F$ y $\beta_{1} \circ b_{i} \leq b_{3}$, para $i=1,2$.

Si $B$ es completo, defina $\mathrm{S}(B)$ como el objeto de los elementos finitos de $B$ tal que

$\left\{b \in B / \forall F \in \Omega^{B}\left(F\right.\right.$ es dirigido $\left.\left.\wedge b \leq \sup _{B}(F) \Rightarrow \exists x \in B(x \in F \wedge b \leq x)\right)\right\}$

Denote por $\mathrm{S}_{B}$ la inclusión de $\mathrm{S}(B)$ en $\Omega^{B}$. Decimos que $b: X \rightarrow B$ es finito si $b$ se factoriza a través de $\mathrm{S}_{B}$. Para todo $X \in|E|, \Omega^{X}$ es un objeto completo, ordenado, con $U_{x}: \Omega^{\Omega^{X}} \rightarrow X$ el adjunto izquierdo de $\downarrow \operatorname{seg}_{\Omega^{X}}$, donde $U_{X}$ es definido por:

$$
\vDash U_{X}(F)=\left\{x \in X / \exists u \in \Omega^{X}(u \in F \wedge x \in u)\right\} .
$$


Definición 1 Un semiretículo con uniones binarias, en un topos elemental E es un objeto $B \in|E|$ parcialmente ordenado con un orden parcial $\leq \subseteq B \times B$ tal que $B$ posee dos operaciones $0: 1 \rightarrow B$ y $v: B \times B \rightarrow B$, con $\omega_{B}: B \rightarrow 1$, el único morfismo de $B \rightarrow 1$ siendo adjunto derecho de $0: 1 \rightarrow B$ y $\triangle_{B}: B \rightarrow$ $B \times B$ la diagonal de $B$ tal que es adjunto derecho de $v: B \times B \rightarrow B$.

Nota. Si $B$ es un semiretículo completo, entonces $\mathrm{S}(B)$ es un semiretículo con uniones binarias de $B$.

Sea $A$ un objeto de $E$, denote por $\mathrm{K}(A)$ el más pequeño subobjeto de $\Omega^{A}$ que contiene a \{\}$_{A}: A \rightarrow \Omega^{A}$ y a $\left\ulcorner\phi_{A}\right\urcorner: 1 \rightarrow \Omega^{A}$ y es cerrado bajo uniones binarias. $A$ es $K$-finito si true $A: 1 \rightarrow \Omega^{A}$ se factoriza a través de $\mathrm{K}(A)$.

Nota. Si $A$ es un objeto de $E$ entonces $\mathrm{S}\left(\Omega^{A}\right)=\mathrm{K}(A)$.

Definición 2 Un objeto $B$ de un topos elemental $y \leq \subseteq B \times B$ tal que $(B, \leq)$ es un objeto ordenado. Decimos que $(B, \leq)$ es retículo algebraico si $(B, \leq)$ es un orden completo tal que el siguiente diagrama conmuta:

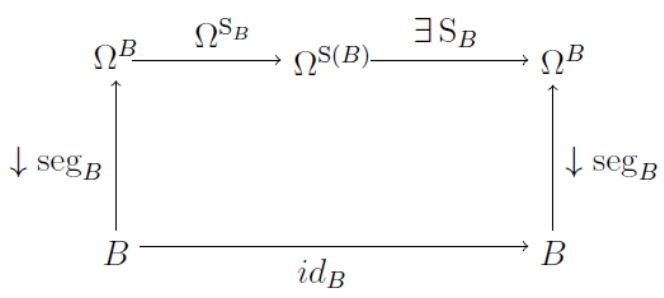

Internamente esto se expresa como

$$
=\forall b \in B, b=\sup _{B}\{a / a \in \mathrm{S}(B) \wedge a \leq b\} .
$$

Para todo $X \in|E|, \Omega^{X}$ es un retículo algebraico ya que \{\}$_{X}: X \rightarrow \Omega^{X}$ está contenido en $\mathrm{S}\left(\Omega^{X}\right)=\mathrm{K}(X)$, y $\mid=Z \in \Omega^{X}, Z=U_{X}\{\{x\} / x \in Z\}$.

Si $V$ es cualquier semiretículo con uniones binarias en un topos elemental $E$, denote por $\mathrm{P}(V)$ al subobjeto de

$$
\left\{V^{\prime} \in \Omega^{V} / V^{\prime} \text { es un ideal de } V\right\} \rightarrow \Omega^{V} .
$$

La fórmula " $V$ ' es un ideal de $V$ " resulta ser:

$\forall v^{\prime} \in V, \forall v \in V\left(\left(v^{\prime} \in V^{\prime} \wedge v \leq v^{\prime} \Rightarrow v \in V^{\prime}\right) \wedge\left(v \in V^{\prime} \wedge v^{\prime} \in V^{\prime} \Rightarrow v \vee v^{\prime} \in V^{\prime}\right)\right)$.

El siguiente Teorema es un resultado de la teoría de retículos probado por Birkhoff [3] y Nachbin [4] y elevado a la teoría de topos. 
Teorema 1 (i) Si $V$ es un semiretículo con uniones binarias en un topos elemental $E, \mathrm{P}(V)$ es un retículo algebraico y $\mathrm{S}(\mathrm{P}(V))=V$.

(ii) Cualquier retículo algebraico a en $E$ es isomorfo a $\mathrm{P}(V)$ con $V$ un semiretículo con uniones en $E$.

Proposición 2 Sea $B$ un objeto completo, ordenado, en un topos E, entonces existe un único morfismo $\mu_{\mathrm{S}(B)}: \mathrm{K}(\mathrm{S}(B)) \rightarrow \mathrm{S}(B)$, tal que el siguiente diagrama conmuta:

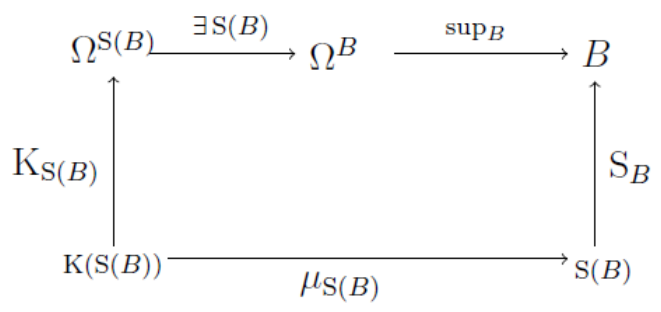

La prueba de esta proposición aparece en [1] (Corolario 1.8).

Proposición 3 Si $X$ es $K$-finito, entonces la fila imagen $\mathrm{Im}: B^{X} \rightarrow \Omega^{B}$ se factoriza a través de $\mathrm{K}(B)$.

Prueba. Sea

$L_{X}=\left\{b \in \Omega^{X} / \forall R \in \Omega^{X \times B}(R\right.$ es funcional $\left.\wedge \operatorname{dom}(R)=b \Rightarrow \operatorname{codom}(R) \in \mathrm{K}(B))\right\}$

(i) \{\}$_{X}: X \rightarrow \Omega^{X}$ se factoriza a través de $L_{X}$.

$\models a \in X \wedge R$ es un funcional $\wedge \operatorname{dom}(R)=\{a\}$

$$
\begin{aligned}
& \Rightarrow \exists ! b \in B,(a, b) \in R \\
& \Rightarrow \exists b \in B,\{(a, b)\}=R \\
& \Rightarrow \operatorname{codom}(R)=\{b\} \wedge \operatorname{dom}(R)=\{a\} \\
& \Rightarrow \operatorname{codom}(R) \in \mathrm{K}(B) .
\end{aligned}
$$

Así tenemos que,

$\models a \in X \Rightarrow \forall R(R$ funcional $\wedge \operatorname{dom}(R)=\{a\} \Rightarrow \operatorname{codom}(R) \in \mathrm{K}(B))$

entonces $=a \in X \Rightarrow\{a\}_{X} \in L_{X}$ y así \{\}$_{X}$ se factoriza a través de $L_{X}$. 
(ii) $\left\ulcorner\phi_{X}\right\urcorner$ se factoriza a través de $L_{X}$.

Probemos que $\mid=R \in \Omega^{X \times B}(\operatorname{dom}(R)=\phi \Rightarrow R=\phi)$ :

$$
\begin{aligned}
\models \operatorname{dom}(R)=\phi & \Leftrightarrow \exists P R_{1}(R)=\phi \\
& \Rightarrow \Omega^{P R_{1}}\left(\exists P R_{1}(R)\right)=\Omega^{P R_{1}}(\phi)=\phi . \\
\left(\exists P R_{1} \vdash \Omega^{P R_{1}}\right) & \Rightarrow R \leq \Omega^{P R_{1}}\left(\exists P R_{1}(R)\right)=\phi \\
& \Rightarrow R=\phi .
\end{aligned}
$$

Así

$$
\begin{aligned}
\models R \text { funcional } \wedge \operatorname{dom}(R)=\phi & \Rightarrow R=\phi \\
& \Rightarrow \operatorname{codom}(R)=\phi \\
& \Rightarrow \operatorname{codom}(R) \in \mathrm{K}(B)
\end{aligned}
$$

entonces

$\forall R\left(R\right.$ funcional $\wedge \operatorname{dom}(R)=\phi \Rightarrow \operatorname{codom}(R) \in \mathrm{K}(B)$ siysolosi $\left.\models\left\ulcorner\phi_{X}\right\urcorner \in L_{X}\right)$.

(iii) $L_{X}$ es cerrado bajo uniones binarias.

Defina $\left.R\right|_{(-)}$como sigue: $|=R|_{a}=\{(\bar{a}, b) / \bar{a} \in a \wedge(\bar{a}, b) \in R\}$. Se tiene que:

$$
\begin{aligned}
& \models a, b \in L_{X} \wedge R \text { es funcional } \wedge \operatorname{dom}(R)=a \cup b \\
& \Rightarrow \quad a,\left.\left.b \in L_{X} \wedge R\right|_{a} \cup R\right|_{b}=\left.R \wedge R\right|_{a} \text { es funcional } \wedge \\
&\left.R\right|_{b} \text { es funcional } \wedge \operatorname{dom}\left(\left.R\right|_{a}\right)=a \wedge \operatorname{dom}\left(\left.R\right|_{b}\right)=b \\
& \Rightarrow \operatorname{codom}\left(\left.R\right|_{a}\right) \in \mathrm{K}(B) \wedge \operatorname{codom}\left(\left.R\right|_{b}\right) \in \mathrm{K}(B) \wedge \\
& \operatorname{codom}\left(\left.\left.R\right|_{a} \cup R\right|_{b}\right)=\operatorname{codom}\left(\left.R\right|_{a} \cup \operatorname{codom}\left(\left.R\right|_{b}\right)\right) \\
& \Rightarrow \operatorname{codom}\left(\left.\left.R\right|_{a} \cup R\right|_{b}\right) \in \mathrm{K}(B) \\
& \Rightarrow \operatorname{codom}(R) \in \mathrm{K}(B) .
\end{aligned}
$$

Entonces $=a, b \in L_{X} \Rightarrow V_{R}(R$ funcional $\wedge \operatorname{dom}(R)=a \cup b \Rightarrow \operatorname{codom}(R) \in$ $\mathrm{K}(B))$ y así $=a, b \in L_{X} \Rightarrow a \cup b \in L_{X}$, y luego por $(i),(i i),($ iii) se tiene que $\mathrm{K}(X) \subseteq L_{X}$. Como $X$ es $K$-finito se tiene que $\ulcorner X\urcorner \in L_{X}$, pero $\ulcorner X\urcorner \in L_{X}$ si y solo si $I m$ se factoriza a través de $\mathrm{K}(B)$. 


\section{Resultado principal}

Teorema 4 Para $X \in|E|$, las siguientes condiciones son equivalentes:

(i) $X$ es $K$-finito.

(ii) Para todo semiretículo con uniones binarias $B$, la diagonal $\triangle_{B}^{X}: B \rightarrow$ $B^{X}$ tiene un adjunto izquierdo.

Prueba. $\quad(i i) \Rightarrow(i) \mathrm{K}(X)$ es un semiretículo con uniones binarias entonces $\triangle_{\mathrm{K}(X)}^{X}: \mathrm{K}(X) \rightarrow \mathrm{K}(X)^{X}$ tiene un adjunto izquierdo $V_{X}$, entonces

$$
\begin{aligned}
\models f \in \mathrm{K}(X)^{X} & \Rightarrow f \leq \triangle_{\mathrm{K}(X)}^{X} \circ V_{X}(f) \\
& \Rightarrow \forall a\left(f(a) \leq \triangle_{\mathrm{K}(X)}^{X}\left(V_{X}(f)(a)\right)\right) \\
& \Rightarrow \forall a\left(f(a) \leq V_{X}(f)\right)
\end{aligned}
$$

entonces

$$
\vDash f \in \mathrm{K}(X)^{X} \Rightarrow\{a \in X / \exists \in X, a \in f(x)\} \subseteq V_{X}(f) .
$$

En particular $\models\{a \in X / \exists x \in X, a \in\{x\}\} \subseteq V_{X}\left(\{\}_{X}\right)$, pero $\{a \in$ $X / \exists x \in X, a=x\}=X$, entonces $\models\ulcorner X\urcorner \subseteq V_{X}\left(\{\}_{X}\right)$ y así tenemos que $\models\ulcorner X\urcorner=V_{X}\left(\{\}_{X}\right)$, por lo que $\ulcorner X\urcorner$ se factoriza a través de $\mathrm{K}(X)$ y entonces $X$ es $K$-finito.

$(i) \Rightarrow(i i)$ Sea $B$ un semiretículo con uniones binarias. Defina $V_{B}^{X}$ la composición $I m \circ \mu_{B}: B^{X} \rightarrow \mathrm{K}(B) \rightarrow B$. Probaremos que $V_{B}^{X}$ es un adjunto izquierdo a la función diagonal $\triangle_{B}^{X}$. Sabemos que el siguiente diagrama conmuta, por el Teorema 1 y la Proposición 2:

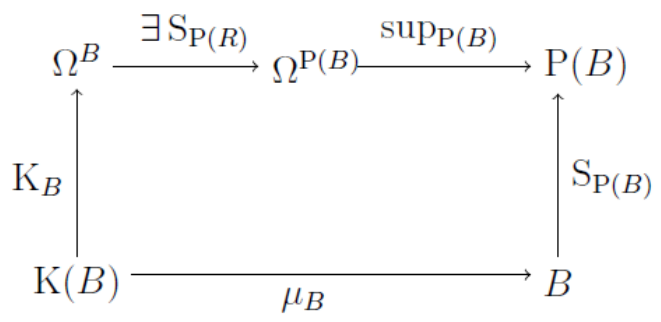

Por tanto, $\models F \in \Omega^{B}, F \in \mathrm{K}(B) \Rightarrow \mu_{B}(F)=\sup _{\mathrm{P}(B)}(F)$. Por otro lado, como $\sup _{\mathrm{P}(B)}$ es el sup para $\mathrm{P}(B)$, tenemos que

$$
=F \in \Omega^{B}, F \in \mathrm{K}(B) \Rightarrow\left(\sup _{\mathrm{P}(B)}(F) \leq a \Leftrightarrow \forall b(b \in F \Rightarrow b \leq a)\right)
$$


y así

$$
\models F \in \Omega^{B}, F \in \mathrm{K}(B) \Rightarrow\left(\mu_{B}(F) \leq a \Leftrightarrow \forall b(b \in F \Rightarrow b \leq a)\right) .
$$

Como $\operatorname{Im}$ se factoriza a través de $\mathrm{K}(B)$, tenemos que $\models f \in B^{X} \Rightarrow \operatorname{Im}(f) \in$ $\mathrm{K}(B)$ por lo que

$$
\models f \in B^{X}\left(u_{B}(\operatorname{Im}(f)) \leq a \Leftrightarrow \forall b(b \in \operatorname{Im}(f) \Rightarrow b \leq a)\right)
$$

y entonces $\mid=f \in B^{X}\left(u_{B}(\operatorname{Im}(f)) \leq a \Leftrightarrow \triangle_{B}^{X}(a)\right)$. Por tanto $\operatorname{Im} \circ u_{B} \vdash \triangle_{B}^{X}$.

De esta forma se obtiene el resultado anunciado.

\section{Referencias}

[1] Acuña-Ortega, O. (1977) Finiteness in Topoi. Ph. D. Dissertation, Wesleyan University, Middletown, CT.

[2] Acuña-Ortega, O. (2011) "Una nota sobre objetos k-finitos en un topos Booleano con el objeto de los números naturales", Revista de Matemática: Teoría y Aplicaciones 19(2): 239-245.

[3] Birkhoff, G. (1973) Lattice Theory, 3rd Ed. American Mathematical Society Colloquium Publications, Volume XXV, Providence RI.

[4] Nachbin, L. (1949) "On a characterization of the lattice of all ideals of a Boolean ring", Fundamenta Mathematicae, 36(1): 137-142. 
